\title{
Local bifurcation of electrohydrodynamic waves on a conducting fluid
}

Zhi Lin, Yi Zhu, and Zhan Wang

Citation: Physics of Fluids 29, 032107 (2017); doi: 10.1063/1.4979064

View online: http://dx.doi.org/10.1063/1.4979064

View Table of Contents: http://aip.scitation.org/toc/phf/29/3

Published by the American Institute of Physics

\section{Articles you may be interested in}

Vortex dynamics in nonlinear free surface flows

Physics of Fluids 29, 032101 (2017); 10.1063/1.4977801

The fate of pancake vortices

Physics of Fluids 29, 031701 (2017); 10.1063/1.4977975

Stability analysis of the rimming flow inside a uniformly heated rotating horizontal cylinder

Physics of Fluids 29, 032102 (2017); 10.1063/1.4977802

Theoretical analysis and simulation of obstructed breakup of micro-droplet in T-junction under an asymmetric pressure difference

Physics of Fluids 29, 032003 (2017); 10.1063/1.4978300

Self-ignition induced by cylindrically imploding shock adapting to a convergent channel

Physics of Fluids 29, 031702 (2017); 10.1063/1.4979135

Topology changes in a water-oil swirling flow

Physics of Fluids 29, 032109 (2017); 10.1063/1.4979277

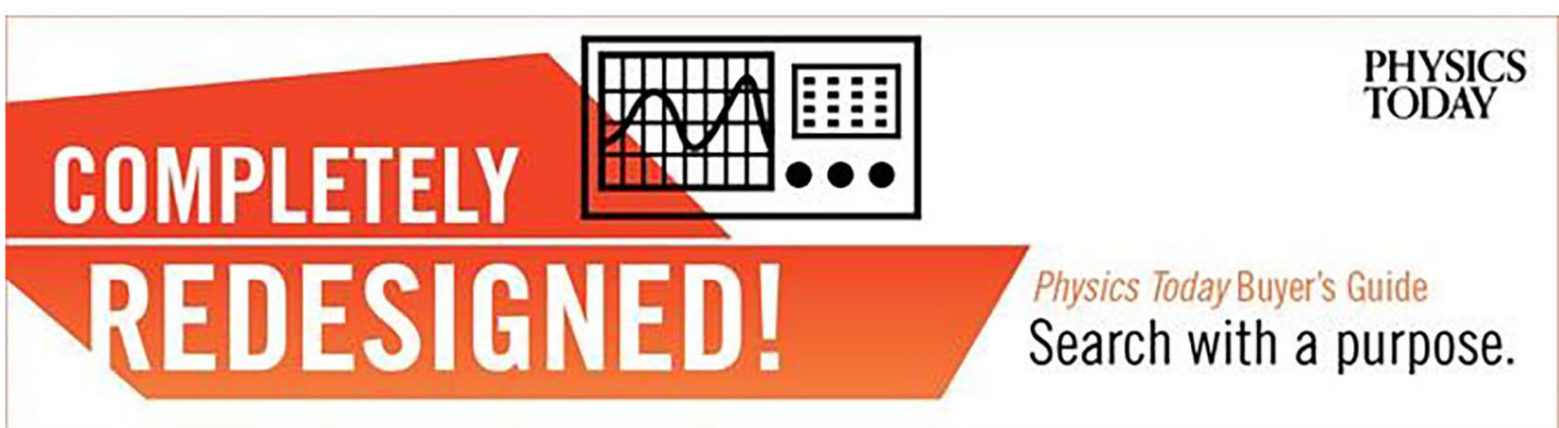




\title{
Local bifurcation of electrohydrodynamic waves on a conducting fluid
}

\author{
Zhi Lin, ${ }^{1}$ Yi Zhu, ${ }^{2}$ and Zhan Wang ${ }^{3,4,5, a)}$ \\ ${ }^{1}$ School of Mathematical Sciences, Zhejiang University, Zhejiang 310027, China \\ ${ }^{2}$ Zhou Pei-Yuan Center for Applied Mathematics, Tsinghua University, Beijing 100084, China \\ ${ }^{3}$ Key Laboratory for Mechanics in Fluid Solid Coupling Systems, Institute of Mechanics, \\ Chinese Academy of Sciences, Beijing 100190, China \\ ${ }^{4}$ School of Engineering Science, University of Chinese Academy Sciences, Beijing 100049, China \\ ${ }^{5}$ Department of Mathematical Sciences, University of Bath, Claverton Down, Bath BA2 7AY, United Kingdom
}

(Received 14 September 2016; accepted 9 March 2017; published online 29 March 2017)

\begin{abstract}
We are concerned with progressive waves propagating on a two-dimensional conducting fluid when a uniform electric field is applied in the direction perpendicular to the undisturbed free surface. The competing effects of gravity, surface tension, and electrically induced forces are investigated using both analytical and numerical techniques for an inviscid and incompressible fluid flowing irrotationally. We simplify the full Euler equations by expanding and truncating the Dirichlet-Neumann operators in the Hamiltonian formulation of the problem. The numerical results show that when the electric parameter is in a certain range, the bifurcation structure near the minimum of the phase speed is rich with Stokes, solitary, generalized solitary, and dark solitary waves. In addition to symmetric solutions, asymmetric solitary waves featuring a multi-packet structure are found to occur along a branch of asymmetric generalized solitary waves that itself bifurcates from Stokes waves of finite amplitude. The detailed bifurcation diagrams, together with typical wave profiles, are presented. Published by AIP Publishing. [http://dx.doi.org/10.1063/1.4979064]
\end{abstract}

\section{INTRODUCTION}

Electrohydrodynamic surface waves, a subject which is concerned with the interplay between an electric field and fluid mechanics, was pioneered by Taylor and Melcher among others in the 1960s. ${ }^{15,16,25,26}$ Modern studies of Electrohydrodynamics (EHD) are driven by its practical significance in chemistry, biology, and industry, involving functional interfaces (adhesives, electrowetting, coatings, etc.), biological membranes, and cooling systems (see Refs. 4, 12 and 13 and the references therein). A comprehensive understanding of the bifurcation mechanism, pattern formation, and dynamics of EHD surface waves is crucial for the success of these new applications.

An applied electric field can exert considerably large forces at electrified interfaces in many situations of practical relevance, ${ }^{16}$ and its orientation with respect to the undisturbed interface plays an important role in the system. The normal electric field, which is imposed perpendicular to the undisturbed interface, has effects to destabilize the liquid-air interface or the interface of two fluids with different permittivities. A typical example is electrocapillary waves under normal electric fields whose dispersion relation reads $\omega^{2}=\left(\sigma k^{3}-\epsilon_{p} E_{0}^{2} k^{2}\right) / \rho$, where $\omega$ is the frequency, $k$ the wavenumber, $\sigma$ the surface tension, $\epsilon_{p}$ the permittivity of the air, $E_{0}$ the strength of the electric field, and $\rho$ the density of the liquid. ${ }^{14}$ It is readily apparent that the electric field can provide energy to a certain range of wavenumbers to induce instability. The tangential electric field, as opposed to the

a) z.wang5@bath.ac.uk normal electric field, has a stabilizing effect on the interface since it provides a dispersive contribution to the linear system. ${ }^{5,22,27}$ It can delay the formation of the film rupture ${ }^{27}$ and even suppress the Rayleigh-Taylor instability. ${ }^{5}$ In the present work, we concentrate on the electrohydrodynamic behavior of a liquid metal in the presence of a strong normal electric field. On physical grounds, this setting is relevant to two particular problems: the so-called "Taylor cone" first described by Taylor ${ }^{25}$ who was primarily interested in the disintegration of water drops in a strong electric field such as thunderstorm, and the instability of the extended horizontal surface of a conducting liquid under the action of a vertical electric field. $^{26}$

In this paper, we study capillary-gravity waves propagating on the surface of a perfect conducting fluid under the influence of a normal electric field in the inviscid setting. The nonelectrical gravity-capillary waves have received considerable attention in the past few decades due to their complexity and applications in wind-ocean coupling. It is well-known that gravity-capillary solitary waves in deep water bifurcate from the minimum of the phase speed and feature oscillatory decaying tails. ${ }^{28,29}$ The underlying mechanism is that the group velocity and the phase velocity are equal at the phase speed minimum, and furthermore at the same point, the associated cubic nonlinear Schrödinger (NLS) equation, which is a weakly nonlinear model governing the dynamics of the envelope, is of focussing type, and hence admits sech-type bright soliton solutions. ${ }^{1}$ For an air-water interface, these wavepacket solitary waves bifurcate from infinitesimal periodic waves with a wavelength of $1.7 \mathrm{~cm}$ and a speed of $23.1 \mathrm{~cm} / \mathrm{s}$. While for an air-mercury interface, the carrier wavelength at the bifurcation point is of approximately $1.2 \mathrm{~cm}$ with the minimum 
phase speed of $19.3 \mathrm{~cm} / \mathrm{s}$. However when the vertical electric field is imposed, the induced net forces on the free surface can turn the associated NLS equation into a defocusing type for a certain range of the electric parameter (see Section III for details), hence disallow wavepacket solitary waves from bifurcating about the uniform state. In view of the above argument, two questions arise naturally when the electric strength is this particular range. The first question is the existence of steady dark solitary waves in the primitive equations, as predicted by the defocusing NLS equation. Dark solitons, which feature biasymptotic uniform states in far fields with a phase jump in between, have been observed and thoroughly investigated in nonlinear optics, plasma physics, and Bose-Einstein condensates. ${ }^{8,24}$ There is a deep analogy between waves in these fields and on the surface of water. In the case of water waves, steady dark solitary wave was first discovered by Bridges and Donaldson. ${ }^{2}$ They found that this type of wave arises through the secondary criticality of Stokes waves in finite depth. Dark solitons on the surface of water were recently observed in a wave tank by Chabchoub et al. ${ }^{3}$ The second question is the existence of bright solitary waves with finite amplitudes in the primitive equations, which are not ruled out by the NLS equation, since the weakly nonlinear theory is valid only at small amplitudes. In the present paper, we give positive answers to both questions based on numerical computations, and the result has potential applications in water waves. Particularly, the existence of steady dark solitary waves indicates that this type of nonlinear waves can be found not only in large-scale gravity waves but also in small-scale gravitycapillary waves using normal electric fields and conducting liquids.

Analytical and numerical investigations on the effect of normal electric fields on inviscid gravity-capillary waves propagating on dielectric or conducting fluids were reported by several groups, most of which were based on long-wave models. Easwaran $^{7}$ and Perel'man ${ }^{23}$ derived the Korteweg-de Vries $(\mathrm{KdV})$ equation and the modified $\mathrm{KdV}$ equation, respectively, for a conducting liquid film in the presence of a vertical electric field. Under the assumption that the electrode is placed laterally far away, Gleeson et al. ${ }^{9}$ derived a new long-wave model with a term involving Hilbert transform, which is called the Benjamin-Ono-KdV equation. In the same paper, when the Bond number is close to $1 / 3$ and the electric field is rather weak, they also derived the fifth-order Benjamin-Ono-KdV equation, which was further explored by Hammmerton and Basso. ${ }^{10}$ Papageorgiou et al. ${ }^{21}$ studied the nonlinear stability of a dielectric liquid film wetting an electrode based on a longwave system to identify the critical electric parameter for the stability exchange.

This paper aims at providing theoretical prediction and numerical evidence for the existence of various solitary waves for conducting fluids under normal electric fields. If we denote by $h$ the thickness of the undisturbed fluid and assume a normal electric field acts with uniform value $E_{0}$ far away, the dispersion relation for the problem reads (see formula (5.155) in Ref. 11)

$$
c_{p}^{2}=\frac{\omega^{2}}{k^{2}}=\tanh (k h)\left(\frac{g}{k}-\frac{\epsilon_{p} E_{0}^{2}}{\rho}+\frac{\sigma}{\rho} k\right),
$$

where $c_{p}$ is called the phase speed and $g$ is the acceleration of gravity. If the fluid is (impure) water or mercury and it is deeper than a few centimetres, $c_{p}$ attains its minimum $c_{\min }$ at a large $k$, which corresponds to a wavelength of 1-2 cm. From the linear theory, we can expect wavepacket solitary waves near $c_{\min }$. At physical grounds, at $c_{\min }$ the group speed and the phase speed coincide, therefore the wavepacket can propagate without changing shape. While near $c_{\min }$, the speed of the carrier wave can be slightly modified by the finite-amplitude effects to match the propagation speed of the envelope. To investigate the bifurcation structure near the phase speed minimum can contribute to designing the lab experiments as well as understanding similar wave phenomena in other physical systems. We will show in Section $\mathrm{V}$ that for a strong electric field, the bifurcation structure near $c_{\min }$ is rich with Stokes, solitary, generalized solitary, and dark solitary waves. Due to the small wavelength of carrier waves near $c_{\min }$ for water and mercury, the deep fluid assumption is a reasonable approximation for gravitycapillary waves under a normal electric field. From now until the end of the paper, we assume that the fluid is of infinite depth.

The organization of the paper is as follows. Following this section, the problem setup and the governing equations are discussed in Section II. We perform the normal-form analysis at $c_{\min }$ in Section III resulting in the cubic nonlinear Schrödinger equation whose type (focusing or defocusing) depends on the electric parameter. Rather than addressing the full Euler and Maxwell equations, we derive a series of computational models resulting from the Taylor expansions of the DirichletNeumann operators in the Hamiltonian formulation of the full problem in Section IV. What follows in Section V is the numerical results on various nonlinear traveling-wave solutions and the bifurcation behavior near $c_{\min }$. Finally concluding remarks are given in Section VI.

\section{GOVERNING EQUATIONS}

We consider an inviscid, incompressible fluid of constant density $\rho$ and of infinite depth. We introduce horizontal coordinates $x$ directed along the undisturbed fluid surface and a vertical coordinate $y$ measured upward from the equilibrium surface. The displacement of the free surface is denoted by $y=\eta(x, t)$, a function of space and time. Assume the flow is irrotational, then we can describe the velocity field $\overrightarrow{\mathbf{v}}$ inside the fluid by a potential function $\phi$ as $\overrightarrow{\mathbf{v}}=\nabla \phi$, where $\nabla$ is the gradient operator. Incompressibility, $\nabla \cdot \overrightarrow{\mathbf{v}}=0$, implies that the velocity potential $\phi$ satisfies Laplace's equation $\nabla^{2} \phi=0$.

An electric field acts along the positive $y$-direction in space with the uniform value $E_{0}$ in the far field. We assume that the fluid is perfectly conducting so that the electric strength is zero within the fluid. The surrounding medium, which occupies the region above the fluid, is assumed dielectric with permittivity $\epsilon_{p}$. The electrostatic limit of Maxwell's equation implies that the induced magnetic fields are negligible, it then follows that the electric field is also irrotational due to Faraday's law. Therefore, we can introduce the voltage potential $V$ such that $\vec{E}=-\nabla V$ and $\nabla^{2} V=0$, where $\vec{E}$ is the electric field above the fluid. 
The boundary conditions at the free surface $y=\eta(x, t)$ are the kinematic condition, continuity of normal stresses, and vanishing of the electric field. The time evolution of $\eta(x, t)$ is governed by the kinematic boundary condition

$$
\eta_{t}=\phi_{y}-\eta_{x} \phi_{x}
$$

Although the voltage potential vanishes at the free surface, the normal component of the electric field at the interface gives rise to a normal stress on the surface. Therefore, the modified Bernoulli's law at $y=\eta(x, t)$ reads $^{21}$

$$
\phi_{t}+\frac{1}{2}|\nabla \phi|^{2}-\frac{\epsilon_{p}}{2 \rho}|\nabla V|^{2}+g \eta-\frac{\sigma}{\rho} \frac{\eta_{x x}}{\left(1+\eta_{x}^{2}\right)^{3 / 2}}=0,
$$

where $\sigma$ is the surface tension coefficient between the fluid and the surrounding medium, and $g$ is the acceleration due to gravity. Under the deep fluid approximation, the potential $\phi$ must satisfy the boundary condition

$$
\phi_{y} \rightarrow 0, \quad \text { as } y \rightarrow-\infty .
$$

In addition, we assume the electrode is placed laterally far away, therefore the electric strength approaches a constant in the far field, ${ }^{9}$

$$
V_{y} \rightarrow E_{0}, \quad \text { as } y \rightarrow+\infty .
$$

For the sake of convenience in the subsequent analyses, we introduce a new voltage potential $W \triangleq V / E_{0}-y$, so that $W$ is a harmonic function with $W_{y} \rightarrow 0$ as $y \rightarrow \infty$. It follows that $W=-\eta$ at $z=\eta$, and the dynamic boundary condition can be rewritten as

$$
\phi_{t}+\frac{1}{2}|\nabla \phi|^{2}-\frac{\widetilde{E}}{2}\left(|\nabla W|^{2}+2 W_{y}\right)+g \eta-\frac{\sigma}{\rho} \frac{\eta_{x x}}{\left(1+\eta_{x}^{2}\right)^{3 / 2}}=0,
$$

where $\widetilde{E} \triangleq \epsilon_{p} E_{0}^{2} / \rho$. Since we retain gravity, surface tension, and electric field effects, we can non-dimensionalize the system by choosing

$$
\left[\frac{\sigma}{\rho g}\right]^{1 / 2},\left[\frac{\sigma}{\rho g^{3}}\right]^{1 / 4},\left[\frac{\sigma^{3}}{\rho^{3} g}\right]^{1 / 4} \text {, and }\left[\frac{\sigma}{\rho g}\right]^{1 / 2}
$$

as reference length, time, velocity potential, and modified voltage potential, respectively. Therefore the dynamic boundary condition (6) can be recast as

$$
\phi_{t}+\frac{1}{2}|\nabla \phi|^{2}-\frac{E_{p}}{2}\left(|\nabla W|^{2}+2 W_{y}\right)+\eta-\frac{\eta_{x x}}{\left(1+\eta_{x}^{2}\right)^{3 / 2}}=0,
$$

where $E_{p} \triangleq \epsilon_{p} E_{0}^{2} / \sqrt{\rho g \sigma}$ is the only parameter in the problem, while the kinematic boundary condition (2) remains the same.

\section{NORMAL FORM ANALYSIS}

The nonlinear Schrödinger (NLS) equation is a universal model appearing in many contexts of science, including water waves, nonlinear optics, Bose-Einstein condensates, etc. Usually it is used to describe the dynamics of the envelope of a monochromatic wave train in the weakly nonlinear regime. In this section, we show that the NLS equation also appears in the context of electrohydrodynamics. Furthermore, we will show in the subsequent analysis that the type of the NLS equation depends on the electric parameter $E_{p}$, therefore we can determine the range of $E_{p}$ where the NLS equation is defocusing and dark solitary waves can be expected, so as to provide a theoretical prediction for numerical simulations of the primitive Euler equations.

To derive the NLS equation, we first assume that $\phi, \eta$, $W$ are all of order $O(\varepsilon)$, and then rewrite the boundary conditions on $y=\eta$ as evaluations on $y=0$, by developing Taylor expansions of $\phi$ and $W$ about $y=0$,

$$
\phi(x, y, t)=\phi(x, 0, t)+\eta \phi_{y}(x, 0, t)+\frac{\eta^{2}}{2} \phi_{y y}(x, 0, t)+O\left(\varepsilon^{4}\right),
$$

$W(x, y, t)=W(x, 0, t)+\eta W_{y}(x, 0, t)+\frac{\eta^{2}}{2} W_{y y}(x, 0, t)+O\left(\varepsilon^{4}\right)$.

This allows us to rewrite the surface boundary conditions $W=-\eta,(2)$ and (8) at $y=0$ as,

$$
\begin{gathered}
W+\eta=-\eta W_{y}-\frac{\eta^{2}}{2} W_{y y}+O\left(\varepsilon^{4}\right) \\
\eta_{t}-\phi_{y}=\left(\eta \phi_{y y}-\eta_{x} \phi_{x}\right)+\left(\frac{\eta^{2}}{2} \phi_{y y y}-\eta \eta_{x} \phi_{x y}\right)+O\left(\varepsilon^{4}\right) \\
\phi_{t}+\eta-\eta_{x x}-E_{p} W_{y}=-\eta \phi_{t y}-\frac{1}{2}\left(\phi_{x}^{2}+\phi_{y}^{2}\right) \\
+\frac{E_{p}}{2}\left(W_{x}^{2}+W_{y}^{2}+2 \eta W_{y y}\right)-\frac{1}{2} \eta^{2} \phi_{t y y} \\
-\left(\eta \phi_{x} \phi_{x y}+\eta \phi_{y} \phi_{y y}\right)-\frac{3}{2} \eta_{x}^{2} \eta_{x x}+\frac{E_{p}}{2} \\
\times\left(2 \eta W_{x} W_{x y}+2 \eta W_{y} W_{y y}+\eta^{2} W_{y y y}\right)+O\left(\varepsilon^{4}\right)
\end{gathered}
$$

Now consider the propagation of a quasi-monochromatic wave oscillating fast in the propagation direction $x$. To proceed, we define $X=\varepsilon x, T=\varepsilon t, \tau=\varepsilon^{2} t$, and choose $e^{i(k x-\omega t)}$ as the carrier wave, where $k$ is the wavenumber and assumed to be positive, and $\omega$ is the frequency. We then seek an asymptotic solution of these equations in the form

$$
\begin{aligned}
W & =\sum_{n=1}^{\infty} \varepsilon^{n}\left(\sum_{j=0}^{n} W_{n j}\left(X-c_{g} T, y, \tau\right) e^{j i \Theta}\right)+\text { c.c. }, \\
\phi & =\sum_{n=1}^{\infty} \varepsilon^{n}\left(\sum_{j=0}^{n} \phi_{n j}\left(X-c_{g} T, y, \tau\right) e^{j i \Theta}\right)+\text { c.c. }, \\
\eta & =\sum_{n=1}^{\infty} \varepsilon^{n}\left(\sum_{j=0}^{n} \eta_{n j}\left(X-c_{g} T, \tau\right) e^{j i \Theta}\right)+\text { c.c. },
\end{aligned}
$$

where $\Theta=k x-\omega t, c_{g}$ is called the group velocity which will be defined later, and "c.c." represents the complex conjugate. Since the fluid is of infinite depth, the term accommodating the mean drift of the wave vanishes. Therefore, the leading order quantities that govern the evolution of the wavepacket are $W_{11} e^{i \Theta}, \phi_{11} e^{i \Theta}$, and $\eta_{11} e^{i \Theta}$. Substituting the ansatz (14) and (15) into Laplace's equation yields, for leading-order terms,

$$
\left(\phi_{11}\right)_{y y}-k^{2} \phi_{11}=0, \quad\left(W_{11}\right)_{y y}-k^{2} W_{11}=0 .
$$


In view of the far-field conditions, the solutions to (17) take the form

$$
\phi_{11}=\widetilde{\phi}_{11}\left(X-c_{g} T, \tau\right) e^{k y}, \quad W_{11}=\widetilde{W}_{11}\left(X-c_{g} T, \tau\right) e^{-k y},
$$

where $\widetilde{\phi}_{11}$ and $\widetilde{W}_{11}$ are arbitrary functions. Substituting (18) and (16) into the boundary conditions (11)-(13), upon collecting the terms of $O(\varepsilon)$, yields

$$
\begin{gathered}
\widetilde{W}_{11}=-\eta_{11}, \quad \widetilde{\phi}_{11}=-\frac{i \omega}{k} \eta_{11}, \\
\omega^{2}=k\left(1-E_{p} k+k^{2}\right), \quad c_{p}^{2}=\frac{\omega^{2}}{k^{2}}=\frac{1}{k}-E_{p}+k,
\end{gathered}
$$

where $c_{p}$ is called the phase speed. It is obvious that $c_{p}^{2}$ attains its minimum $2-E_{p}$ when $k=1$, and it immediately follows that the problem is linearly well-posed for $E_{p} \leqslant 2$. Solving Laplace's equations for the next order gives

$$
\phi_{20}=\widetilde{\phi}_{20}, \quad \phi_{21}=e^{k y}\left[\widetilde{\phi}_{21}-i y\left(\widetilde{\phi}_{11}\right)_{X}\right], \quad \phi_{22}=e^{2 k y} \widetilde{\phi}_{22},
$$

$$
W_{20}=\widetilde{W}_{20}, W_{21}=e^{-k y}\left[\widetilde{W}_{21}+i y\left(\widetilde{W}_{11}\right)_{X}\right], \quad W_{22}=e^{-2 k y} \widetilde{W}_{22} \text {, }
$$

where all the functions with tilde depend on $X-c_{g} T$ and $\tau$. Inserting these solutions into the conditions on the free surface yields the following:

- Terms in $\varepsilon^{2} e^{0 i \Theta}$ give $\eta_{20}=0$ and $\widetilde{W}_{20}=-2 k\left|\eta_{11}\right|^{2}$.

- Terms in $\varepsilon^{2} e^{i \Theta}$ give the expression of the group velocity

$$
c_{g}=\frac{1-2 E_{p} k+3 k^{2}}{2 \omega}=\omega^{\prime},
$$

and the relations between $\eta_{21}, \widetilde{W}_{21}$, and $\widetilde{\phi}_{21}$ through the following expressions

$$
\widetilde{W}_{21}=-\eta_{21}, \quad \widetilde{\phi}_{21}=\frac{1}{k}\left[-i \omega \eta_{21}+i\left(\widetilde{\phi}_{11}\right)_{X}-c_{g}\left(\eta_{11}\right)_{X}\right] \text {. }
$$

- Terms in $\varepsilon^{2} e^{2 i \Theta}$ result in a system of three linear equations with $\eta_{22}, \widetilde{W}_{22}$, and $\widetilde{\phi}_{22}$ as unknowns. Solving this linear system yields

$$
\begin{aligned}
& \eta_{22}=\frac{-\omega^{2}+E_{p} k^{2}}{2 k^{2}-1} \eta_{11}^{2}, \quad \widetilde{W}_{22}=-\eta_{22}-k \eta_{11}^{2}, \\
& \widetilde{\phi}_{22}=-\frac{i \omega}{k} \eta_{22}+i \omega \eta_{11}^{2} .
\end{aligned}
$$

At the third order, it suffices to consider the fundamental mode $e^{i \Theta}$. Using the method of multiple scales again in Laplace's equations, one obtains

$$
\begin{aligned}
& W_{31}=e^{-k y}\left[\widetilde{W}_{31}+i y\left(\widetilde{W}_{21}\right)_{X}+\frac{y^{2}}{2}\left(\eta_{11}\right)_{X X}\right], \\
& \phi_{31}=e^{k y}\left[\widetilde{\phi}_{31}-i y\left(\widetilde{\phi}_{21}\right)_{X}+\frac{i \omega}{2 k} y^{2}\left(\eta_{11}\right)_{X X}\right] .
\end{aligned}
$$

A considerable amount of algebra on the boundary conditions eventually results in solvability conditions for the fundamental mode that yield the governing equation, the cubic nonlinear
Schrödinger equation, for the envelope $\eta_{11}$. We state the result without tedious calculations,

$$
i\left(\eta_{11}\right)_{\tau}+\alpha\left(\eta_{11}\right)_{X X}+\gamma\left|\eta_{11}\right|^{2} \eta_{11}=0
$$

where $\alpha$ and $\gamma$ take the form

$$
\alpha=\frac{3 k-E_{p}-c_{g}^{2}}{2 \omega}, \quad \gamma=\frac{k}{2 \omega}\left[\frac{2\left(E_{p} k^{2}-\omega^{2}\right)^{2}}{2 k^{2}-1}-2 k^{2}-\frac{1}{2} k^{4}\right] .
$$

Evaluating these coefficients at the minimum of the phase speed $\left(k^{*}=1\right.$ and $\left.\omega^{*}=\sqrt{2-E_{p}}\right)$ yields

$$
\alpha=\frac{1}{2 \sqrt{2-E_{p}}}, \quad \gamma=\frac{1}{\sqrt{2-E_{p}}}\left[4\left(1-E_{p}\right)^{2}-\frac{5}{4}\right] .
$$

It is easy to check that the coefficient $\gamma$ is positive for $E_{p} \in[0,1-\sqrt{5} / 4) \cup(1+\sqrt{5} / 4,2)$, and $\gamma$ is negative if $E_{p} \in(1-\sqrt{5} / 4,1+\sqrt{5 / 4})$. For positive $\gamma$, the NLS equation (28) is of focusing type and hence has the bright soliton solutions

$$
\eta_{11}(X, \tau)=\sqrt{\frac{2 \lambda}{\gamma}} \operatorname{sech}\left(X \sqrt{\frac{\lambda}{\alpha}}\right) e^{i \lambda \tau}
$$

with a positive parameter $\lambda$. Combining (31) with the ansatz (16) gives the leading-order approximation of the wave profile

$$
\begin{aligned}
\eta \approx & \varepsilon \sqrt{\frac{8 \lambda}{\gamma}} \operatorname{sech}\left[\varepsilon\left(x-c_{\min } t\right) \sqrt{\frac{\lambda}{\alpha}}\right] \\
& \times \cos \left[\left(x-c_{\min } t+\varepsilon^{2} \lambda t^{2}\right)+x_{0}\right],
\end{aligned}
$$

where $x_{0}$ is an arbitrary constant determining the initial phase shift between the carrier wave and the envelope. Despite the arbitrariness of the initial phase shift, only two branches of solitary waves have been found to bifurcate from infinitesimal periodic waves in the full Euler equations (Vanden-Broeck and $\left.\operatorname{Dias}^{29}\right)$ : the elevation branch $\left(x_{0}=0\right)$ and the depression branch $\left(x_{0}=\pi\right)$. The other case, with $\gamma$ negative, is the defocusing NLS which admits steady dark soliton solutions characterized by a constant amplitude at infinity and a local dip in the center,

$$
\eta_{11}(X, \tau)=\sqrt{\frac{\lambda}{\gamma}} \tanh \left(X \sqrt{-\frac{\lambda}{2 \alpha}}\right) e^{i \lambda \tau},
$$

where $\lambda$ is an arbitrary negative parameter. Combining (16) and (33) furnishes the approximation of the dark solitary-wave solution in the full Euler equations

$$
\begin{aligned}
\eta \approx & 2 \varepsilon \sqrt{\frac{\lambda}{\gamma}} \tanh \left[\varepsilon\left(x-c_{\min } t\right) \sqrt{-\frac{\lambda}{2 \alpha}}\right] \\
& \times \cos \left[\left(x-c_{\min } t+\varepsilon^{2} \lambda t\right)+x_{0}\right] .
\end{aligned}
$$

It is noted that choosing the initial phase $x_{0}=\pi / 2$ and $3 \pi / 2$ gives symmetric solutions.

\section{DIRICHLET-NEUMANN OPERATORS}

In fiber optics, the NLS equation is usually used ad initio, whereas in water waves, it only represents a reduced model 
whose accuracy and rationality should be verified against solutions of the full Euler equations.

To design a numerical method for the Euler equations, we first introduce the Hamiltonian formulation for the full problem. The total energy of the system is given by the sum of kinetic energy and potential energy,

$$
\begin{aligned}
\mathcal{H}= & \frac{1}{2} \int_{-\infty}^{\infty} \int_{-\infty}^{\eta}|\nabla \phi|^{2} d y d x-\frac{E_{p}}{2} \int_{-\infty}^{\infty} \int_{\eta}^{\infty}|\nabla W|^{2} d y d x \\
& +\frac{1}{2} \int_{-\infty}^{\infty} \eta^{2} d x+\int_{-\infty}^{\infty}\left(\sqrt{1+\eta_{x}^{2}}-1\right) d x
\end{aligned}
$$

where the potential energy consists of three parts in this context: electric potential energy, gravitational potential energy, and surface energy. Following Craig and Sulem ${ }^{6}$ and Zakharov, ${ }^{33}$ we define the value of the velocity potential at the free surface as $\xi(x, t) \triangleq \phi(x, \eta(x, t), t)$, then the divergence theorem yields

$\mathcal{H}=\frac{1}{2} \int_{-\infty}^{\infty}\left[\xi G^{-}(\eta) \xi-E_{p} \eta G^{+}(\eta) \eta+\eta^{2}+2\left(\sqrt{1+\eta_{x}^{2}}-1\right)\right] d x$.

This is the Hamiltonian formulation of the present problem, and written in terms of the Dirichlet-Neumann operators $G^{ \pm}(\eta)$ which are defined as

$$
\begin{gathered}
G^{-}(\eta) \xi \triangleq \phi_{y}-\left.\eta_{x} \phi_{x}\right|_{y=\eta}=\nabla \phi \cdot \mathbf{n} \sqrt{1+\eta_{x}^{2}}, \\
G^{+}(\eta)(-\eta) \triangleq \eta_{x} W_{x}-\left.W_{y}\right|_{y=\eta}=\nabla W \cdot(-\mathbf{n}) \sqrt{1+\eta_{x}^{2}},
\end{gathered}
$$

where $\mathbf{n}$ is the unit normal vector pointing upwards. The Dirichlet-Neumann operator returns the normal derivative at the free surface by solving Laplace's equation with the Dirichlet boundary condition. Following (6), the Dirichlet-Neumann operators can be formally written as Taylor series

$$
G^{-}(\eta)=\sum_{n=0}^{\infty} G_{n}(\eta), \quad G^{+}(\eta)=\sum_{n=0}^{\infty}(-1)^{n} G_{n}(\eta),
$$

where $G_{n}(\eta)$ takes the following form: for $n \geqslant 1$,

$$
G_{n}(\eta)=-G_{0}^{n-1} \partial_{x} \frac{\eta^{n}}{n !} \partial_{x}-\sum_{j=1}^{n} G_{0}^{j} \frac{\eta^{j}}{j !} G_{n-j}(\eta)
$$

and $G_{0}=\left(-\partial_{x x}\right)^{1 / 2}$. Substituting (39) into (36) yields

$$
\mathcal{H}[\eta, \xi]=\widetilde{\mathcal{H}}_{m}[\eta, \xi]+O\left(\xi^{2} \eta^{m+1}, \eta^{m+3}\right),
$$

where $\widetilde{\mathcal{H}}_{m}$ takes the form

$$
\begin{aligned}
\widetilde{\mathcal{H}}_{m}= & \frac{1}{2} \int_{-\infty}^{\infty}\left[\xi \sum_{n=0}^{m} G_{n}(\eta) \xi-E_{p} \eta \sum_{n=0}^{m}(-1)^{n} G_{n}(\eta) \eta+\eta^{2}\right. \\
& \left.+2\left(\sqrt{1+\eta_{x}^{2}}-1\right)\right] d x
\end{aligned}
$$

Disregarding terms of order $O\left(\xi^{2} \eta^{m+1}, \eta^{m+3}\right)$, the evolution equations for $\xi$ and $\eta$ can be obtained by taking variational derivatives of the approximated Hamiltonian $\widetilde{\mathcal{H}}_{m}$ as

$$
\eta_{t}=\frac{\delta \widetilde{\mathcal{H}}_{m}}{\delta \xi}, \quad \xi_{t}=-\frac{\delta \widetilde{\mathcal{H}}_{m}}{\delta \eta}
$$

We list below the models for $m \leqslant 4$ with tedious calculations omitted:

$$
\begin{gathered}
\eta_{t}-G_{0} \xi=\sum_{n=1}^{m} G_{n}(\eta) \xi \\
\xi_{t}+\left(1-E_{p} G_{0}-\partial_{x x}\right) \eta=\sum_{n=2}^{m+1} \mathcal{N}_{n}-\partial_{x}\left[\frac{\eta_{x}^{3}}{\left(1+\sqrt{1+\eta_{x}^{2}}\right) \sqrt{1+\eta_{x}^{2}}}\right],
\end{gathered}
$$

where the nonlinear terms $\mathcal{N}_{n}$ are given as

$$
\begin{aligned}
& \mathcal{N}_{2}=\frac{1}{2}\left[\left(G_{0} \xi\right)^{2}-\xi_{x}^{2}\right]+\frac{E_{p}}{2}\left[\left(G_{0} \eta\right)^{2}-\eta_{x}^{2}-2 G_{1} \eta\right], \\
& \mathcal{N}_{3}=\left(G_{0} \xi\right)\left(G_{1} \xi+\eta_{x} \xi_{x}\right)-E_{p}\left[\left(G_{0} \eta\right)\left(G_{1} \eta+\eta_{x}^{2}\right)-G_{2} \eta\right],
\end{aligned}
$$

$$
\begin{aligned}
\mathcal{N}_{4}= & \frac{1}{2}\left[\left(G_{1} \xi+\eta_{x} \xi_{x}\right)^{2}+2\left(G_{0} \xi\right)\left(G_{2} \xi\right)-\eta_{x}^{2}\left(G_{0} \xi\right)^{2}\right] \\
& +\frac{E_{p}}{2}\left[\left(G_{1} \eta+\eta_{x}^{2}\right)^{2}+2\left(G_{0} \eta\right)\left(G_{2} \eta\right)-\eta_{x}^{2}\left(G_{0} \eta\right)^{2}-2 G_{3} \eta\right],
\end{aligned}
$$

$$
\begin{aligned}
\mathcal{N}_{5}= & \left(G_{0} \xi\right)\left(G_{3} \xi\right)+\left(G_{2} \xi-\eta_{x}^{2} G_{0} \xi\right)\left(G_{1} \xi+\eta_{x} \xi_{x}\right) \\
& -E_{p}\left[\left(G_{0} \eta\right)\left(G_{3} \eta\right)+\left(G_{2} \eta-\eta_{x}^{2} G_{0} \eta\right)\left(G_{1} \eta+\eta_{x}^{2}\right)-G_{4} \eta\right] .
\end{aligned}
$$

Here, we suppress the dependence on $\eta$ in $G_{j}(\eta)$ for notational simplicity. The efficiency and accuracy of these numerical models for nonelectric surface water waves (i.e., $E_{p}=0$ ) were discussed by Wang and Milewski ${ }^{17}$ and Nicholls and Reitich. ${ }^{20}$

\section{NUMERICAL RESULTS}

In order to study the pattern formation or progressive waves in more details in free-surface water waves, we should consider the problems in the primitive equations or quantitative models, which may show a rich variety of bifurcations for fluid phenomena. In this section, based on the quantitative model with $m=4$ in (44) and (45), we study the local bifurcation near the minimum of the phase speed. The rationale for a quintic truncation (i.e., $m=4$ ) is to guarantee the same local bifurcation behaviour between the model and full potential flow. That is because for small $|\gamma|$, under a new rescaling, the modulations of wavepackets should be well described by a cubic-quintic NLS equation. In order to derive a correct cubic-quintic NLS equation, the quintic truncation is necessary (the reader is referred to Ref. 30 for more details).

When $E_{p} \in[0,1-\sqrt{5} / 4) \cup(1+\sqrt{5} / 4,2)$, the associated NLS is focusing, therefore the local bifurcation mechanism is analogous to that of the gravity-capillary waves in deep water (i.e., $E_{p}=0$ ): two branches of wavepacket solitary waves (elevation and depression branches) bifurcate from infinitesimal periodic waves at $c_{\min }$. An immediate question arising from the aforementioned discussion is what is the bifurcation mechanism near $c_{\min }$ when the associated NLS equation is of 
defocusing type (i.e., $\left.E_{p} \in(1-\sqrt{5} / 4,1+\sqrt{5} / 4)\right)$. To investigate this problem, we confine ourselves to the defocusing case and choose $E_{p}=0.6$ in subsequent computations.

\section{A. Dark solitary waves}

First of all, we consider the steady dark solitary waves in systems (44) and (45), whose existence has already been predicted by the envelope equation: the defocusing NLS equation. We assume that waves propagate with velocity $c$, then it follows that $\eta$ and $\xi$ depend on $x-c t$ only, namely,

$$
\begin{gathered}
-c \eta_{\theta}-G_{0} \xi=\sum_{n=1}^{m} G_{n}(\eta) \xi, \\
-c \xi_{\theta}+\left(1-E_{p} G_{0}-\partial_{\theta \theta}\right) \eta=\sum_{n=1}^{m} \mathcal{N}_{n}-\partial_{\theta} \\
\times\left[\frac{\eta_{\theta}^{3}}{\left(1+\sqrt{1+\eta_{\theta}^{2}}\right) \sqrt{1+\eta_{\theta}^{2}}}\right],
\end{gathered}
$$

where $\theta=x-c t$. Pseudo-spectral method is used in all numerical experiments. We expand the unknowns $\eta$ and $\xi$ as

$$
\eta=\sum_{n=-N}^{N} a_{n} e^{2 \pi i n \theta / L}, \quad \xi=\sum_{n=-N}^{N} b_{n} e^{2 \pi i n \theta / L},
$$

with $a_{n}=a_{-n}$ real and $b_{n}=-b_{-n}$ pure imaginary for symmetric waves, where $L$ is the length of the computational domain which is sufficiently long and carefully chosen for different numerical experiments. We substitute (52) into (50) and (51), evaluate at uniformly distributed grid points, project them onto the same wavenumber, and use Newton's method to solve for unknowns $a_{n}$ and $b_{n} \cdot{ }^{31}$ It is noted that the derivatives and differential operator $G_{0}$ are easy to calculate via Fourier multipliers. We choose (34) as the initial guess of the Newton algorithm, where the small parameter $\varepsilon$ can be obtained by noticing

$$
c_{\min }-\varepsilon^{2} \lambda \approx c \Longrightarrow \varepsilon \approx \sqrt{\frac{c-c_{\min }}{\lambda}} .
$$

Near the bifurcation point $c_{\min }$, dark solitary waves are characterized by Stokes waves on the tails. Noticing that the dark soliton solution (33) adds a phase shift of $\pi$ to the carrier wave, we choose a domain $L=(2 n+1) \pi$ for a considerably large $n$. This numerical trick has been successfully used to search for symmetric dark solitary waves in other nonlinear waves problems such as hydroelastic waves ${ }^{19}$ and interfacial gravity-capillary waves. $^{31}$

Two branches of dark solitary were found in systems (50) and (51). They are, respectively, the depression branch with a local minimum at the center and the elevation branch featuring a local maximum at the center. The bifurcation diagram of the depression branch, together with typical profiles, is presented in Figure 1. It shows that dark solitary waves bifurcate from infinitesimal periodic waves, and along the speed-amplitude curve there is a turning point at $c \approx 1.1898$, beyond which the wave profiles feature two solitary pulses with non-decaying tails. Further following the bifurcation curve, ripples in the far field vanish as $c$ becomes smaller than $c_{\min }$, therefore multihump solitary waves appear. The dashed curves in Figures 1(a) and 1(b) are the predictions of the defocusing NLS equation given by (33), which show good agreement at small amplitudes. In Figure 1(b), the dotted curves were obtained for $n$ $=31$, and it turns out that four Stokes waves were added in the far field on both sides without any noticeable changes of the profiles already obtained for $n=25$ (solid curve). This fact strongly indicates that as the domain size increases, more and more periodic waves can be added, therefore we can numerically obtain true dark solitary waves as the domain approaches infinity. The elevation branch is shown in Figure 2 , and its bifurcation mechanism is similar to the depression branch.


FIG. 1. Dark solitary waves with positive curvature at the center. (a) Speed-amplitude bifurcation diagram near the minimum of the phase speed computed based on the quintic model (solid curve), together with the NLS prediction (dashed curve). (b) From top to bottom: typical wave profiles corresponding to points $1 a$, $1 b, 1 c, 1 d$ labeled in the left panel, respectively. As the amplitude increases, dark solitary waves becomes two-pulse generalised solitary waves, and finally turn to bright solitary waves as the speed reaches the subcritical regime. For dark solitary waves ( $1 a$ and $1 b)$, solutions were generated in the computational domains of $51 \pi$ (solid curve) and $67 \pi$ (dotted curve), respectively, and the NLS prediction (dashed curve) is also shown for reference. 



FIG. 2. Dark solitary waves with negative curvature at the center. (a) Speed-amplitude bifurcation diagram obtained by solving the quintic model (solid curve), together with the NLS prediction (dashed curve). (b) From top to bottom: typical wave profiles corresponding to points $2 a, 2 b, 2 c, 2 d$ labeled in the left panel, respectively. As the amplitude increases, dark solitary waves becomes two-pulse generalised solitary waves, and finally turn to bright solitary waves as the speed reaches the subcritical regime. For dark solitary waves $2 a$ and $2 b$, solutions were generated in the computational domains of $51 \pi$ (solid curve) and $67 \pi$ (dotted curve), respectively, and the NLS prediction (dashed curve) is also shown for reference.

\section{B. Bright and generalized solitary waves}

Though the NLS approximation implies that there are no small-amplitude bright solitons for $E_{p} \in(1-\sqrt{5} / 4,1+\sqrt{5} / 4)$, the normal form analysis does not rule out the existence of moderate-amplitude solitary waves in our Hamiltonian model. The existence of multi-hump bright solitary waves shown in the first experiment also inspires us to seek single-hump fully localized solutions in the same system.

For $E_{p}=0$, it is well documented ${ }^{29}$ that deep-water gravity-capillary bright solitary waves bifurcate from infinitesimal periodic waves since the associated NLS equation is of focusing type (i.e., $\gamma=11 \sqrt{2} / 8$ in (28)) at $c_{\min }$. We started our numerical experiment from searching for bright solitary waves in systems (50) and (51) for $E_{p}=0$ with initial guess given by (32), then gradually increased the value of $E_{p}$ as a parameter in the numerical continuation scheme, and finally stopped at $E_{p}=0.6$. We found that bright solitary waves do exist in our problem for relatively large $E_{p}$ even though the associated NLS equation is defocusing. However, the bifurcation mechanism of these wavepacket solitary waves is novel, since they do not bifurcate from infinitesimal periodic waves. If we follow the bifurcation curve by increasing $c$, as the curve crosses the minimum of the phase speed, the corresponding waves grow up to the length of the entire computational domain and become generalized solitary waves due to the resonance with periodic waves. Further along the curve, the branch of generalized solitary waves joins the branch of Stokes waves. This bifurcation mechanism was found in both depression branch (Figure 3(a)) and elevation branch (Figure 4(a)), and typical wave profiles are presented in Figures 3(b) and 4(b), respectively. (a)

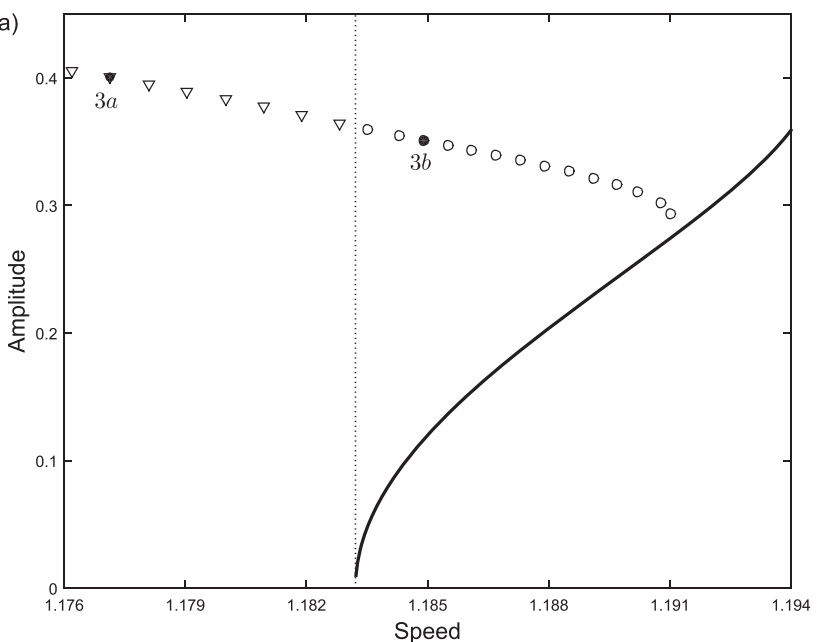

(b)


FIG. 3. Bright and generalized solitary waves of depression. (a) Bright solitary waves (downward-pointing triangle) appear along the branch of generalized solitary waves (circles) that itself bifurcates from periodic waves (solid curve) of finite amplitude. (b) Top: typical profile of depression solitary waves (corresponding to the point $3 a$ labeled in the left figure); bottom: typical profile of generalized solitary waves of depression (corresponding to the point $3 b$ shown in the left figure), which is generated in the computational domains of $62 \pi$ (solid curve) and $50 \pi$ (dotted curve). 



FIG. 4. Bright and generalized solitary waves of elevation. (a) Bright solitary waves (upward-pointing triangle) appear along the branch of two-pulse generalized solitary waves (circles) that itself bifurcates from periodic waves (solid curve). (b) Top: typical profile of elevation solitary waves (corresponding to the point $4 a$ labeled in the left figure); bottom: typical profile of generalized solitary waves of elevation (corresponding to the point $4 b$ shown in the left figure), which is generated in the computational domains of $70 \pi$ (solid curve) and $62 \pi$ (dotted curve).

The generalized solitary waves were computed in different domains $(50 \pi$ and $62 \pi$ for depression waves, and $62 \pi$ and $70 \pi$ for elevation waves), and it turns out that the profiles generated in a smaller domain are almost exactly on the top of the profiles for the larger domain. This fact strongly suggests the existence of true generalized solitary waves, though we carried out the numerical experiments in periodic domains. We should emphasize that the computations of generalized solitary waves are very sensitive to the size of the domain, which selects a particular periodicity, and we only chose $L=2 n \pi$ therefore the branch originates from the fundamental branch of Stokes waves. It is noted that similar solutions and bifurcation diagram to those shown in Figure 3 have been found for depression hydroelastic waves. ${ }^{18}$

\section{Asymmetric solitary waves}

Finally, we consider the existence of asymmetric traveling waves for the problem. It was numerically found by Wang

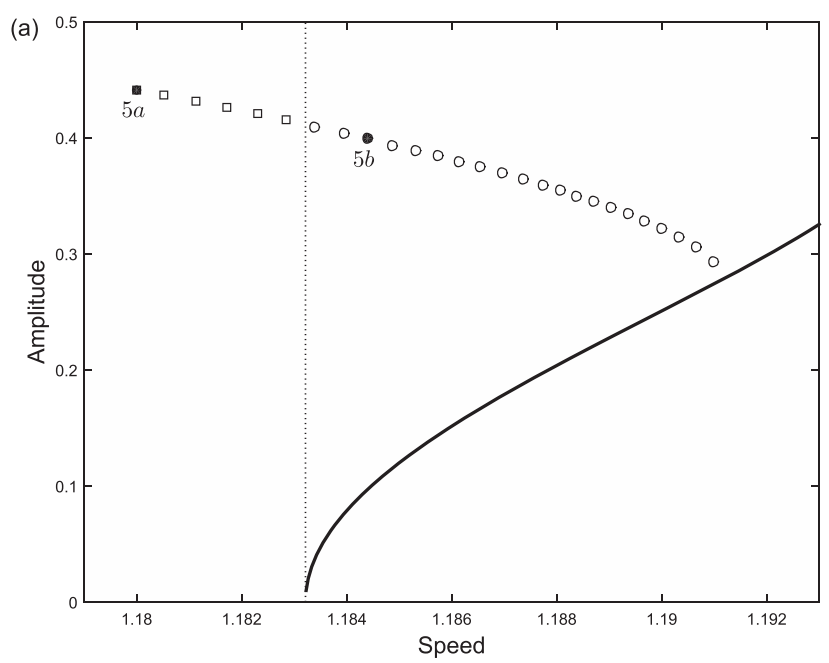

et al. ${ }^{32}$ that asymmetric gravity-capillary solitary waves exist below $c_{\min }$ in deep water in the full Euler equations. These authors also showed that the solutions feature a multi-packet structure and appear via spontaneous symmetry-breaking bifurcations. Motivated by this work, we try to test the existence of asymmetric solitary wave under a normal electric field and investigate how the electric force alters the bifurcation structure.

Similar to the second experiment, we started from the solution obtained for nonelectric gravity-capillary waves (i.e., $E_{p}=0$ ), treated $E_{p}$ as a continuation parameter, and finally stopped the computation at $E_{p}=0.6$. An asymmetric solitary wave was then obtained, which was composed of a depression wave and an elevation one separated by small ripples (see Figure 5(b)). Once one solution is found, the bifurcation branch near $c_{\min }$ can be completed by fixing the value of $E_{p}$ and varying the speed of the wave. It was found that in contrast to the case without electric field, asymmetric solitary waves do (b)
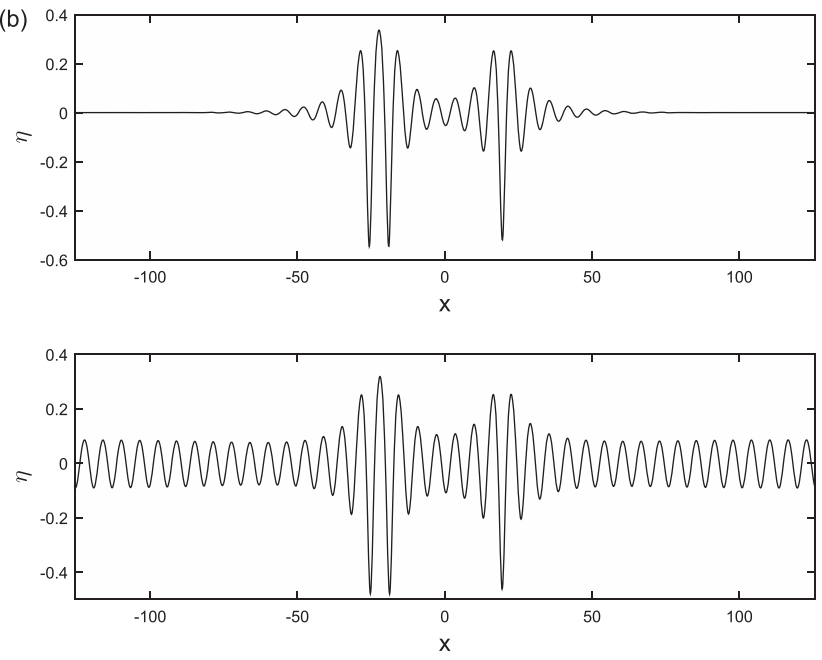

FIG. 5. Asymmetric bright and generalized solitary waves. (a) Asymmetric bright solitary waves (squares) appear along a branch of asymmetric generalized solitary waves (circles) that itself bifurcates from symmetric periodic waves (solid curve) of finite amplitude. (b) shows the typical profiles for a bright solitary wave (top, $5 a$ ) and a generalized solitary wave (bottom, $5 b$ ). 
not bifurcate from the branch of symmetric solitary waves. Instead, as one increases the translating speed, asymmetric solitary waves become asymmetric generalized solitary waves for $c>c_{\min }$. As the speed is further increased, it terminates on the branch of finite-amplitude Stokes waves (see Figure 5). To the best of our knowledge, it was the first time that asymmetric generalized solitary waves were found in water-wave problems.

\section{CONCLUSION}

In the present paper, we have investigated twodimensional gravity-capillary solitary waves propagating on a conducting fluid under a vertical electric field. We simplified the full Euler equations by expanding and truncating the Dirichlet-Neumann operator and developed a Hamiltonian model which was used to study the local bifurcation mechanism near the minimum of the phase speed. It has been shown that for relatively large electric parameter where the associated NLS equation is of defocusing type, dark solitary waves bifurcate from infinitesimal periodic waves and exist at supercritical speeds. As the amplitude increases, the speed-amplitude bifurcation curve experiences a limiting point, beyond which the waves turn to be generalized solitary waves with two solitary pulses. As one further reduces the speed, bright solitary waves featuring two depression waves placed side-by-side appear for $c<c_{\text {min }}$. We have also shown that though the NLS equation is defocusing, bright solitary waves (including single-hump symmetric waves and multi-hump asymmetric waves) exist at finite amplitudes, but the bifurcation mechanism is novel, since they appear along the branches of generalized solitary waves that themselves bifurcate from the branch of Stokes waves.

Numerical evidence presented in the paper provides a possible way to find steady dark solitary waves in small-scale gravity-capillary waves, while the associated NLS equation gives some guidance on the magnitude of the electric fields to be used in the lab experiments. On the numerical side, the discovery of the rich bifurcation structure near the phase speed minimum in the truncated Hamiltonian model leads us to postulate the similar phenomenon in the full equations for water waves, which is of great interest for further research. The existence of new steady solutions also inspires us to study their stability and dynamic properties in the future.

\section{ACKNOWLEDGMENTS}

This work was initiated while Z.W. visited Zhejiang University and Tsinghua University. He wishes to acknowledge the support of University of Bath in the form of an International Funding Scheme, and the hospitality of the Department of Mathematics at Zhejiang University and Zhou Pei-Yuan Center for Applied Mathematics at Tsinghua University. This work was supported by National Natural Science Foundation of China (Grant Nos. 11232012, 11201419, J1210038, and 51408141), Key Research Program of Frontier Sciences, CAS (Grant No. QYZDB-SSW-SYS015), and Strategic
Priority Research Program of the Chinese Academy of Sciences (Grant No. XDB22040203).

${ }^{1}$ T. R. Akylas, "Envelope solitons with stationary crests," Phys. Fluids A 5(4), 789-791 (1993).

${ }^{2}$ T. J. Bridges and N. M. Donaldson, "Secondary criticality of water waves. Part I. Definition, bifurcation and solitary waves," J. Fluid Mech. 565, 381-417 (2006).

${ }^{3}$ A. Chabchoub, O. Kimmoun, H. Branger, N. Hoffmann, D. Proment, M. Onorato, and N. Akhmediev, "Experimental observation of dark solitons on the surface of water," Phys. Rev. Lett. 110, 124101 (2013).

${ }^{4}$ X. P. Chen, J. S. Cheng, and X. Z. Yin, "Advances and applications of electrohydrodynamics," Chin. Sci. Bull. 48(11), 1055-1063 (2003).

${ }^{5}$ R. Cimpeanu, D. T. Papageorgiou, and P. G. Petropoulos, "On the control and suppression of the Rayleigh-Taylor instability using electric fields," Phys. Fluids 26, 022105 (2014).

${ }^{6}$ W. Craig and C. Sulem, "Numerical simulation of gravity waves," J. Comput. Phys. 108, 73-83 (1993).

${ }^{7}$ C. V. Easwaran, "Solitary waves on a conducting fluid layer," Phys. Fluids 31, 3442-3443 (1988).

${ }^{8}$ G. Fibich, The Nonlinear Schrödinger Equation: Singular Solutions and Optical Collapse (Springer, 2015).

${ }^{9}$ H. Gleeson, P. W. Hammerton, D. T. Papageorgiou, and J.-M. Vanden-Broeck, "A new application of the Korteweg-de Vries BenjaminOno equation in interfacial electrohydrodynamics,” Phys. Fluids 19, 031703 (2007).

${ }^{10}$ P. W. Hammerton and A. P. Basso, "The effect of a normal electric field on wave propagation on a fluid film," Phys. Fluids 26, 012107 (2014).

${ }^{11}$ M. Hunter, Linear and Nonlinear Free Surface Flows in Electrohydrodynamics (University College London, 2013).

${ }^{12}$ R. K. Jain and C. Maldarelli, "Stability of thin viscoelastic films with applications to biological membrane deformation," Ann. N. Y. Acad. Sci. 404, 89-102 (1983).

${ }^{13}$ S. F. Kistler and P. M. Schweizer, Stability of Thin Viscoelastic Films with Applications to Biological Membrane Deformation (Chapman and Hall, London, 1997).

${ }^{14}$ L. D. Landau and E. M. Lifshits, Electrodynamics of Continuous Media (Pergamon Press, 1984).

15 J. R. Melcher, Field-Coupled Surface Waves (MIT Press, 1963).

${ }^{16}$ J. R. Melcher and G. I. Taylor, "Electrohydrodynamics: A review of the role of interfacial shear stresses," Annu. Rev. Fluid Mech. 1, 111-146 (1969).

${ }^{17}$ P. A. Milewski and Z. Wang, "Transversally periodic solitary gravitycapillary waves,” Proc. R. Soc. A 470, 20130537 (2014).

${ }^{18}$ P. A. Milewski, J.-M. Vanden-Broeck, and Z. Wang, "Hydroelastic solitary waves in deep water," J. Fluid Mech. 679, 628-640 (2011).

${ }^{19}$ P. A. Milewski, J.-M. Vanden-Broeck, and Z. Wang, "Steady dark solitary flexural gravity waves," Proc. R. Soc. A 469, 20120485 (2013).

${ }^{20}$ D. P. Nicholls and F. Reitich, "Stability of high-order perturbative methods for the computation of Dirichlet-Neumann operators," J. Comput. Phys. 170, 276-298 (2001).

${ }^{21}$ D. T. Papageorgiou, P. G. Petropoulos, and J.-M. Vanden-Broeck, "Gravity capillary waves in fluid layer under normal electric field," Phys. Rev. E 72, 051601 (2005).

${ }^{22}$ D. T. Papageorgiou and J.-M. Vanden-Broeck, "Large amplitude capillary waves in electrified fluid sheets," J. Fluid Mech. 508, 71-88 (2004).

${ }^{23}$ T. L. Perel'man, A. Kh. Fridman, and M. M. El'yashevich, "A modified Korteweg-de Vries equation in electrohydrodynamics,” Zh. Elesp. Teor. Fiz. 66, 1316-1323 (1974), see http://jetp.ac.ru/cgi-bin/dn/e_039_04_0643.pdf.

${ }^{24}$ V. N. Serkin and A. Hasegawa, "Novel soliton solutions of the nonlinear Schrödinger equation model," Phys. Rev. Lett. 85, 4502-4505 (2000).

${ }^{25} \mathrm{G}$. I. Taylor, "Disintegration of water droplets in an electric field," Proc. R. Soc. A 280, 383-397 (1964).

${ }^{26}$ G. I. Taylor and A. D. McEwan, "The stability of a horizontal fluid interface in a vertical electric field," J. Fluid Mech. 22, 1-15 (1965).

${ }^{27}$ B. S. Tilley, P. G. Petropoulos, and D. T. Papageorgiou, "Dynamics and rupture of planar electrified liquid sheets," Phys. Fluids 13, 3547-3563 (2001).

${ }^{28}$ J.-M. Vanden-Broeck, Gravity-Capillary Free-Surface Flows (Cambridge University Press, 2010).

${ }^{29}$ J.-M. Vanden-Broeck and F. Dias, "Gravity-capillary solitary waves in water of infinite depth and related free-surface flows," J. Fluid Mech. 240, 549-557 (1992). 
${ }^{30}$ Z. Wang, P. A. Milewski, and J.-M. Vanden-Broeck, "Computation of threedimensional flexural-gravity solitary waves in arbitrary depth," Procedia IUTAM 11, 119-129 (2014).

${ }^{31}$ Z. Wang, J.-M. Vanden-Broeck, and H. Meng, "A quasi-planar model for gravity-capillary interfacial waves in deep water," Stud. Appl. Math. 133, 232-256 (2014).
${ }^{32}$ Z. Wang, J.-M. Vanden-Broeck, and P. A. Milewski, "Asymmetric gravity-capillary solitary waves on deep water," J. Fluid Mech. 759, R2 (2014).

${ }^{33}$ V. E. Zakharov, "Stability of periodic waves of finite amplitude on the surface of a deep fluid," J. Appl. Mech. Tech. Phys. 9, 190-194 (1968). 